\title{
SUCCESSIVE ITERATION AND POSITIVE SOLUTIONS FOR A $p$-LAPLACIAN MULTIPOINT BOUNDARY VALUE PROBLEM
}

\author{
BO SUN ${ }^{凶 1}$, XIANGKUI ZHAO ${ }^{2}$ and WEIGAO GE ${ }^{3}$
}

(Received 10 March, 2007; revised 28 April, 2008)

\begin{abstract}
In this paper, we study the existence of positive solutions for the one-dimensional $p$-Laplacian differential equation,

$$
\left(\phi_{p}\left(u^{\prime}(t)\right)\right)^{\prime}+q(t) f\left(t, u(t), u^{\prime}(t)\right)=0, \quad t \in(0,1),
$$

subject to the multipoint boundary condition

$$
u^{\prime}(0)=\sum_{i=1}^{n} \alpha_{i} u^{\prime}\left(\xi_{i}\right), \quad u(1)=\sum_{i=1}^{n} \beta_{i} u\left(\xi_{i}\right),
$$

by applying a monotone iterative method.

2000 Mathematics subject classification: 34B10, 34B18.

Keywords and phrases: successive iteration; positive solutions; multipoint boundary value problems; $p$-Laplacian.
\end{abstract}

\section{Introduction}

In this paper, we will consider the positive solutions to the $p$-Laplacian boundary value problem

$$
\begin{array}{r}
\left(\phi_{p}\left(u^{\prime}(t)\right)\right)^{\prime}+q(t) f\left(t, u(t), u^{\prime}(t)\right)=0, \quad t \in(0,1), \\
u^{\prime}(0)=\sum_{i=1}^{n} \alpha_{i} u^{\prime}\left(\xi_{i}\right), \quad u(1)=\sum_{i=1}^{n} \beta_{i} u\left(\xi_{i}\right),
\end{array}
$$

\footnotetext{
${ }^{1}$ Department of Mathematics, Beijing Institute of Technology, Beijing 100081, PR China; e-mail: sunbo19830328@163.com.

${ }^{2}$ Applied Science School, University of Science and Technology Beijing, Beijing 100083, PR China.

${ }^{3}$ Department of Mathematics, Beijing Institute of Technology, Beijing 100081, PR China; e-mail: gew@bit.edu.cn.

(c) Australian Mathematical Society 2008, Serial-fee code 0334-2700/08
} 
where $\phi_{p}(s)=|s|^{p-2} s$ with $p>1, \xi_{i} \in(0,1)$ with $0<\xi_{1}<\xi_{2}<\cdots<\xi_{n}<1$, and $\alpha_{i}, \beta_{i}, f, q$ satisfy:

(H1) $0 \leq \alpha_{i}, \beta_{i}<1(i=1,2, \ldots, n)$ satisfy $0 \leq \sum_{i=1}^{n} \alpha_{i}, \sum_{i=1}^{n} \beta_{i}<1$;

(H2) $f(t, x, y) \in C([0,1] \times[0,+\infty) \times R \rightarrow[0,+\infty)), q(t)$ is a nonnegative continuous function defined on $(0,1)$ and $q(t) \not \equiv 0$ on any subinterval of $(0,1)$. In addition, $\int_{0}^{1} q(t) d t<+\infty$.

Here, a positive solution of (1.1) and (1.2) means a solution $u^{*}$ satisfying $u^{*}(t)>0$, $0<t<1$.

The existence and multiplicity of positive solutions for linear and nonlinear multipoint boundary value problems have been widely studied by many authors (see $[1-6,8]$ and the references therein). However, there are not many papers which are concerned with the computational methods of these problems. Then the question arises: "How can we find the solutions when their existence is known?"

More recently, when under the assumption that $f$ is allowed to depend only on $t$ and $u$ but not on $u^{\prime}$, Ma et al. [7] proved the existence of positive solutions of a multipoint $p$-Laplacian boundary value problem via a monotone iterative technique.

So, motivated by all the works above, we investigate here the iteration and existence of positive solutions for the multipoint boundary value problem with $p$-Laplacian (1.1) and (1.2), which will extend all the previous research. We do not require the existence of lower and upper solutions. By applying monotone iterative techniques, we construct some successive iterative schemes to approximate the solutions in this paper.

\section{Preliminaries}

In this section, we give the preliminaries and some definitions.

Definition 2.1. Let $E$ be a real Banach space. A nonempty closed set $P \subset E$ is said to be a cone provided that:

- $a u+b v \in P$ for all $u, v \in P$ and all $a \geq 0, b \geq 0$; and

- $u,-u \in P$ implies $u=0$.

Definition 2.2. The map $\alpha$ is said to be concave on $[0,1]$, if

$$
\alpha(t u+(1-t) v) \geq t \alpha(u)+(1-t) \alpha(v)
$$

for all $u, v \in[0,1]$ and $t \in[0,1]$.

Let $E$ be the Banach space $C^{1}[0,1]$ endowed with the norm

$$
\|u\|:=\max \left\{\max _{0 \leq t \leq 1}|u(t)|, \max _{0 \leq t \leq 1}\left|u^{\prime}(t)\right|\right\} .
$$

We denote $E_{+}=C_{+}^{1}[0,1]=\{u \in E \mid u(t) \geq 0, t \in[0,1]\}$, and define the cone $P \subset E$ by

$$
P=\{u \in E \mid u(t) \geq 0, u \text { is concave and nonincreasing on }[0,1]\} .
$$

Throughout, it is assumed that (H1) and (H2) hold. 
Lemma 2.1. Suppose that $y \in C^{1}[0,1]$ with $\left(\phi_{p}\left(y^{\prime}(t)\right)\right)^{\prime} \in L^{1}[0,1]$ satisfies

$$
\begin{gathered}
-\left(\phi_{p}\left(y^{\prime}(t)\right)\right)^{\prime} \geq 0, \quad t \in(0,1), \\
y^{\prime}(0)=\sum_{i=1}^{n} \alpha_{i} y^{\prime}\left(\xi_{i}\right), \quad y(1)=\sum_{i=1}^{n} \beta_{i} y\left(\xi_{i}\right) .
\end{gathered}
$$

Then, $y(t)$ is concave and $y(t) \geq 0, y^{\prime}(t) \leq 0$ on $[0,1]$, that is, $y \in P$.

The proof is very easy since $0 \leq \sum_{i=1}^{n} \alpha_{i}, \sum_{i=1}^{n} \beta_{i}<1$, so we omit it here.

For all $x \in C_{+}^{1}[0,1]$, suppose that $u$ is a solution of the problem (1.1) and (1.2). Then

$$
\begin{aligned}
u^{\prime}(t) & =\phi_{p}^{-1}\left(A_{x}-\int_{0}^{t} q(s) f\left(s, x(s), x^{\prime}(s)\right) d s\right), \\
u(t) & =B_{x}-\int_{t}^{1} \phi_{p}^{-1}\left(A_{x}-\int_{0}^{s} q(r) f\left(r, x(r), x^{\prime}(r)\right) d r\right) d s,
\end{aligned}
$$

where $A_{x}, B_{x}$ satisfy the boundary conditions, that is,

$$
\begin{aligned}
\phi_{p}^{-1}\left(A_{x}\right) & =\sum_{i=1}^{n} \alpha_{i} \phi_{p}^{-1}\left(A_{x}-\int_{0}^{\xi_{i}} q(s) f\left(s, x(s), x^{\prime}(s)\right) d s\right), \\
B_{x} & =\sum_{i=1}^{n} \beta_{i}\left[B_{x}-\int_{\xi_{i}}^{1} \phi_{p}^{-1}\left(A_{x}-\int_{0}^{s} q(r) f\left(r, x(r), x^{\prime}(r)\right) d r\right) d s\right] .
\end{aligned}
$$

Hence,

$$
\begin{aligned}
u(t)= & -\frac{\sum_{i=1}^{n} \beta_{i} \int_{\xi_{i}}^{1} \phi_{p}^{-1}\left(A_{x}-\int_{0}^{s} q(r) f\left(r, x(r), x^{\prime}(r)\right) d r\right) d s}{1-\sum_{i=1}^{n} \beta_{i}} \\
& -\int_{t}^{1} \phi_{p}^{-1}\left(A_{x}-\int_{0}^{s} q(r) f\left(r, x(r), x^{\prime}(r)\right) d r\right) d s
\end{aligned}
$$

where $A_{x}$ satisfies (2.1).

Lemma 2.2. For all $x \in C_{+}^{1}[0,1]$, there exists a unique $A_{x}$ with

$$
A_{x} \in\left[-\frac{\phi_{p}\left(\sum_{i=1}^{n} \alpha_{i}\right)}{1-\phi_{p}\left(\sum_{i=1}^{n} \alpha_{i}\right)} \int_{0}^{1} q(s) f\left(s, x(s), x^{\prime}(s)\right) d s, 0\right]
$$

satisfying (2.1).

The proof is similar to [7, proof of Lemma 2.2], so we omit it here.

For any $x \in C_{+}^{1}[0,1]$, let $A_{x}$ be the unique constant satisfying (2.1) corresponding to $x$. Then we have the following lemma. 
LEMMA 2.3. $A_{x}: C_{+}^{1}[0,1] \rightarrow R$ has the following properties:

(a) $A_{x}: C_{+}^{1}[0,1] \rightarrow R$ is continuous about $x$;

(b) if $f(t, x, y)$ is nondecreasing about $x$ and nonincreasing about $y$ on $[0,1] \times$ $[0,+\infty) \times(-\infty, 0]$, then $A_{x}$ is nonincreasing on $P$.

The proof is similar to [7, proof of Lemma 2.3], so we omit it here.

\section{Main results}

For notational convenience, we denote

$$
A=\frac{\left(1-\sum_{i=1}^{n} \beta_{i}\right) \phi_{p}^{-1}\left(1-\phi_{p}\left(\sum_{i=1}^{n} \alpha_{i}\right)\right)}{\left(1-\sum_{i=1}^{n} \beta_{i} \xi_{i}\right) \phi_{p}^{-1}\left(\int_{0}^{1} q(s) d s\right)} .
$$

We will prove the following existence results.

THEOREM 3.1. Assume that (H1) and (H2) hold, and there exists a $>0$ such that:

(S1) $f\left(t, x_{1}, y_{1}\right) \leq f\left(t, x_{2}, y_{2}\right)$ for any $0 \leq t \leq 1,0 \leq x_{1} \leq x_{2} \leq a,-a \leq y_{2} \leq y_{1}$ $\leq 0$

(S2) $\max _{0 \leq t \leq 1} f(t, a,-a) \leq \phi_{p}(a A)$;

(S3) $f(t, 0,0) \not \equiv 0$ for $0 \leq t \leq 1$.

Then the boundary value problem (1.1) and (1.2) has two positive nonincreasing, concave solutions $w^{*}$ and $v^{*}$ such that

$$
\begin{aligned}
& \quad 0<w^{*} \leq a, \quad-a \leq\left(w^{*}\right)^{\prime} \leq 0 \\
& \qquad \text { and } \lim _{n \rightarrow \infty} w_{n}=\lim _{n \rightarrow \infty} T^{n} w_{0}=w^{*}, \quad \lim _{n \rightarrow \infty}\left(w_{n}\right)^{\prime}=\lim _{n \rightarrow \infty}\left(T^{n} w_{0}\right)^{\prime}=\left(w^{*}\right)^{\prime}, \\
& \text { where } w_{0}(t)=a-a t \frac{1-\sum_{i=1}^{n} \beta_{i}}{1-\sum_{i=1}^{n} \beta_{i} \xi_{i}}, \quad 0 \leq t \leq 1,
\end{aligned}
$$

and

$$
\begin{aligned}
& \quad 0<v^{*} \leq a, \quad-a \leq\left(v^{*}\right)^{\prime} \leq 0 \\
& \qquad \begin{array}{l}
\text { and } \lim _{n \rightarrow \infty} v_{n}=\lim _{n \rightarrow \infty} T^{n} v_{0}=v^{*}, \quad \lim _{n \rightarrow \infty}\left(v_{n}\right)^{\prime}=\lim _{n \rightarrow \infty}\left(T^{n} v_{0}\right)^{\prime}=\left(v^{*}\right)^{\prime}, \\
\text { where } v_{0}(t)=0, \quad 0 \leq t \leq 1,
\end{array}
\end{aligned}
$$

where

$$
\begin{aligned}
(T u)(t)= & -\frac{\sum_{i=1}^{n} \beta_{i} \int_{\xi_{i}}^{1} \phi_{p}^{-1}\left(A_{u}-\int_{0}^{s} q(r) f\left(r, u(r), u^{\prime}(r)\right) d r\right) d s}{1-\sum_{i=1}^{n} \beta_{i}} \\
& -\int_{t}^{1} \phi_{p}^{-1}\left(A_{u}-\int_{0}^{s} q(r) f\left(r, u(r), u^{\prime}(r)\right) d r\right) d s .
\end{aligned}
$$


The iterative schemes in Theorem 3.1 are

$$
\begin{gathered}
w_{0}(t)=a-a t \frac{\left(1-\sum_{i=1}^{n} \beta_{i}\right)}{\left(1-\sum_{i=1}^{n} \beta_{i} \xi_{i}\right)}, \quad w_{n+1}=T w_{n}=T^{n} w_{0}, \quad n=0,1,2 \ldots \\
\text { and } \quad v_{0}(t)=0, \quad v_{n+1}=T v_{n}=T^{n} v_{0}, \quad n=0,1,2 \ldots
\end{gathered}
$$

They start off with a known linear function and the zero function, respectively.

Proof. We define an operator $T: P \rightarrow E$ by (3.1). Then, from the definition of $T$, we deduce that, for each $u \in P$, there exists $T u \in C^{1}[0,1]$ which is nonnegative and satisfies (1.2). Moreover, by Lemma 2.1 we have that $T u$ is concave, that is, $T u \in P$, and $(T u)^{\prime}(t) \leq 0$ on $[0,1]$. So, $T: P \rightarrow P$.

The continuity of $T$ is obvious. Now, we prove that $T$ is compact. Let $\Omega \subset P$ be a bounded set. It is easy to prove that $T(\Omega)$ is bounded and equicontinuous. Then the Arzelà-Ascoli theorem guarantees that $T \Omega$ is relatively compact, which means that $T$ is compact. Then, $T: P \rightarrow P$ is completely continuous, and each fixed point of $T$ in $P$ is a solution of (1.1) and (1.2).

For any $u_{i} \in P(i=1,2)$ with $u_{1} \leq u_{2}$ and $u_{1}^{\prime} \geq u_{2}^{\prime}$, let $A_{u_{i}}(i=1,2)$ be two constants decided in (2.1) corresponding to $u_{i} \in P(i=1,2)$, then by (S1) and Lemma 2.3 we have $A_{u_{1}} \geq A_{u_{2}}$. From the definition of $T$, we can easily get $T u_{1} \leq$ $T u_{2}$.

We denote

$$
\bar{P}_{a}=\{u \in P \mid\|u\| \leq a\}
$$

Then, in what follows, we first prove that $T: \bar{P}_{a} \rightarrow \bar{P}_{a}$. If $u \in \bar{P}_{a}$, then $\|u\| \leq a$, and

$$
\begin{gathered}
0 \leq u(t) \leq u(0)=\max _{0 \leq t \leq 1}|u(t)| \leq\|u\| \leq a, \\
-a \leq-\|u\| \leq-\max _{0 \leq t \leq 1}\left|u^{\prime}(t)\right|=u^{\prime}(1) \leq u^{\prime}(t) \leq 0 .
\end{gathered}
$$

So by (S1) and (S2)

$0 \leq f\left(t, u(t), u^{\prime}(t)\right) \leq f(t, a,-a) \leq \max _{0 \leq t \leq 1} f(t, a,-a) \leq \phi_{p}(a A), \quad$ for $0 \leq t \leq 1$

In fact,

$$
\begin{aligned}
\|T u\| & =\max \left\{\max _{0 \leq t \leq 1}|(T u)(t)|, \max _{0 \leq t \leq 1}\left|(T u)^{\prime}(t)\right|\right\} \\
& =\max \left\{(T u)(0),-(T u)^{\prime}(1)\right\} .
\end{aligned}
$$


By (3.1) and Lemma 2.2,

$$
\begin{aligned}
(T u)(0) & {\left[\sum_{i=1}^{n} \beta_{i} \int_{\xi_{i}}^{1} \phi_{p}^{-1}\left(A_{u}-\int_{0}^{s} q(r) f\left(r, u(r), u^{\prime}(r)\right) d r\right) d s\right]\left[1-\sum_{i=1}^{n} \beta_{i}\right]^{-1} } \\
& -\int_{0}^{1} \phi_{p}^{-1}\left(A_{u}-\int_{0}^{s} q(r) f\left(r, u(r), u^{\prime}(r)\right) d r\right) d s \\
\leq & {\left[\sum _ { i = 1 } ^ { n } \beta _ { i } \int _ { \xi _ { i } } ^ { 1 } \phi _ { p } ^ { - 1 } \left(\frac{\phi_{p}\left(\sum_{i=1}^{n} \alpha_{i}\right)}{1-\phi_{p}\left(\sum_{i=1}^{n} \alpha_{i}\right)} \int_{0}^{1} q(s) f\left(s, u(s), u^{\prime}(s)\right) d s\right.\right.} \\
& \left.\left.+\int_{0}^{1} q(r) f\left(r, u(r), u^{\prime}(r)\right) d r\right) d s\right]\left[1-\sum_{i=1}^{n} \beta_{i}\right]^{-1} \\
& +\int_{0}^{1} \phi_{p}^{-1}\left(\frac{\phi_{p}\left(\sum_{i=1}^{n} \alpha_{i}\right)}{1-\phi_{p}\left(\sum_{i=1}^{n} \alpha_{i}\right)} \int_{0}^{1} q(s) f\left(s, u(s), u^{\prime}(s)\right) d s\right. \\
& \left.+\int_{0}^{1} q(r) f\left(r, u(r), u^{\prime}(r)\right) d r\right) d s \\
\leq & {\left[\left(1-\sum_{i=1}^{n} \beta_{i} \xi_{i}\right) \phi_{p}^{-1}\left(\int_{0}^{1} q(s) f\left(s, u(s), u^{\prime}(s)\right) d s\right)\right] } \\
& \times\left[\left(1-\sum_{i=1}^{n} \beta_{i}\right) \phi_{p}^{-1}\left(1-\phi_{p}\left(\sum_{i=1}^{n} \alpha_{i}\right)\right)\right]^{-1} \\
\leq & a A\left[\left(1-\sum_{i=1}^{n} \beta_{i} \xi_{i}\right) \phi_{p}^{-1}\left(\int_{0}^{1} q(s) d s\right)\right] \\
& \times\left[\left(1-\sum_{i=1}^{n} \beta_{i}\right) \phi_{p}^{-1}\left(1-\phi_{p}\left(\sum_{i=1}^{n} \alpha_{i}\right)\right)\right]^{-1} \\
= & a,
\end{aligned}
$$

and

$$
\begin{aligned}
-(T u)^{\prime}(1)= & \phi_{p}^{-1}\left(-A_{u}+\int_{0}^{1} q(s) f\left(s, u(s), u^{\prime}(s)\right) d s\right) \\
\leq & \phi_{p}^{-1}\left(\frac{\phi_{p}\left(\sum_{i=1}^{n} \alpha_{i}\right)}{1-\phi_{p}\left(\sum_{i=1}^{n} \alpha_{i}\right)} \int_{0}^{1} q(s) f\left(s, u(s), u^{\prime}(s)\right) d s\right. \\
& \left.+\int_{0}^{1} q(s) f\left(s, u(s), u^{\prime}(s)\right) d s\right) \\
\leq & a A \frac{\phi_{p}^{-1}\left(\int_{0}^{1} q(s) d s\right)}{\phi_{p}^{-1}\left(1-\phi_{p}\left(\sum_{i=1}^{n} \alpha_{i}\right)\right)} \leq a .
\end{aligned}
$$


Thus, we obtain that

$$
\|T u\|=\max \left\{(T u)(0),-(T u)^{\prime}(1)\right\} \leq a .
$$

Hence, we assert that $T: \bar{P}_{a} \rightarrow \bar{P}_{a}$.

Let

$$
w_{0}(t)=a-a t \frac{1-\sum_{i=1}^{n} \beta_{i}}{1-\sum_{i=1}^{n} \beta_{i} \xi_{i}}, \quad 0 \leq t \leq 1,
$$

so then $w_{0}(t) \in \bar{P}_{a}$. Let $w_{1}=T w_{0}$, so then $w_{1} \in \bar{P}_{a}$. We denote $w_{n+1}=T w_{n}=$ $T^{n} w_{0}, n=0,1,2 \ldots$ Since $T: \bar{P}_{a} \rightarrow \bar{P}_{a}$, we have $w_{n} \in T \bar{P}_{a} \subseteq \bar{P}_{a}, n=1,2, \ldots$ Since $T$ is completely continuous, we assert that $\left\{w_{n}\right\}_{n=1}^{\infty}$ is a sequentially compact set.

Since

$$
\begin{aligned}
w_{1}(t)= & T w_{0}(t) \\
= & -\left[\sum_{i=1}^{n} \beta_{i} \int_{\xi_{i}}^{1} \phi_{p}^{-1}\left(A_{w_{0}}-\int_{0}^{s} q(r) f\left(r, w_{0}(r), w_{0}^{\prime}(r)\right) d r\right) d s\right] \\
& \times\left[1-\sum_{i=1}^{n} \beta_{i}\right]^{-1}-\int_{t}^{1} \phi_{p}^{-1}\left(A_{w_{0}}-\int_{0}^{s} q(r) f\left(r, w_{0}(r), w_{0}^{\prime}(r)\right) d r\right) d s \\
\leq & {\left[\sum _ { i = 1 } ^ { n } \beta _ { i } \int _ { \xi _ { i } } ^ { 1 } \phi _ { p } ^ { - 1 } \left(\frac{\phi_{p}\left(\sum_{i=1}^{n} \alpha_{i}\right)}{1-\phi_{p}\left(\sum_{i=1}^{n} \alpha_{i}\right)} \int_{0}^{1} q(s) f\left(s, w_{0}(s), w_{0}^{\prime}(s)\right) d s\right.\right.} \\
& \left.\left.+\int_{0}^{1} q(r) f\left(r, w_{0}(r), w_{0}^{\prime}(r)\right) d r\right) d s\right]\left[1-\sum_{i=1}^{n} \beta_{i}\right]^{-1} \\
& +\int_{t}^{1} \phi_{p}^{-1}\left(\frac{\phi_{p}\left(\sum_{i=1}^{n} \alpha_{i}\right)}{1-\phi_{p}\left(\sum_{i=1}^{n} \alpha_{i}\right)} \int_{0}^{1} q(s) f\left(s, w_{0}(s), w_{0}^{\prime}(s)\right) d s\right. \\
& \left.+\int_{0}^{1} q(r) f\left(r, w_{0}(r), w_{0}^{\prime}(r)\right) d r\right) d s \\
\leq & a A \frac{\left(1-\sum_{i=1}^{n} \beta_{i} \xi_{i}\right) \phi_{p}^{-1}\left(\int_{0}^{1} q(s) d s\right)}{\left(1-\sum_{i=1}^{n} \beta_{i}\right) \phi_{p}^{-1}\left(1-\phi_{p}\left(\sum_{i=1}^{n} \alpha_{i}\right)\right)}-t A a \frac{\phi_{p}^{-1}\left(\int_{0}^{1} q(s) d s\right)}{\phi_{p}^{-1}\left(1-\phi_{p}\left(\sum_{i=1}^{n} \alpha_{i}\right)\right)} \\
= & a-a t \frac{1-\sum_{i=1}^{n} \beta_{i}}{1-\sum_{i=1}^{n} \beta_{i} \xi_{i}}=w_{0}(t), \quad 0 \leq t \leq 1,
\end{aligned}
$$


and

$$
\begin{aligned}
w_{1}^{\prime}(t)= & \left(T w_{0}\right)^{\prime}(t) \\
= & \phi_{p}^{-1}\left(A_{w_{0}}-\int_{0}^{t} q(s) f\left(s, w_{0}(s), w_{0}^{\prime}(s)\right) d s\right) \\
\geq & \phi_{p}^{-1}\left(-\frac{\phi_{p}\left(\sum_{i=1}^{n} \alpha_{i}\right)}{1-\phi_{p}\left(\sum_{i=1}^{n} \alpha_{i}\right)} \int_{0}^{1} q(s) f\left(s, w_{0}(s), w_{0}^{\prime}(s)\right) d s\right. \\
& \left.-\int_{0}^{t} q(s) f\left(s, w_{0}(s), w_{0}^{\prime}(s)\right) d s\right) \\
\geq & \phi_{p}^{-1}\left(-\frac{1}{1-\phi_{p}\left(\sum_{i=1}^{n} \alpha_{i}\right)} \int_{0}^{1} q(s) f\left(s, w_{0}(s), w_{0}^{\prime}(s)\right) d s\right) \\
\geq & -a A \frac{\phi_{p}^{-1}\left(\int_{0}^{1} q(s) d s\right)}{\phi_{p}^{-1}\left(1-\phi_{p}\left(\sum_{i=1}^{n} \alpha_{i}\right)\right)} \\
= & -a \frac{1-\sum_{i=1}^{n} \beta_{i}}{1-\sum_{i=1}^{n} \beta_{i} \xi_{i}}=w_{0}^{\prime}(t), \quad 0 \leq t \leq 1,
\end{aligned}
$$

so

$$
\begin{gathered}
w_{2}(t)=T w_{1}(t) \leq T w_{0}(t)=w_{1}(t), \quad 0 \leq t \leq 1, \\
w_{2}^{\prime}(t)=\left(T w_{1}\right)^{\prime}(t) \geq\left(T w_{0}\right)^{\prime}(t)=w_{1}^{\prime}(t), \quad 0 \leq t \leq 1 .
\end{gathered}
$$

Hence, by the induction,

$$
w_{n+1} \leq w_{n}, \quad w_{n+1}^{\prime}(t) \geq w_{n}^{\prime}(t), \quad 0 \leq t \leq 1, n=0,1,2 \ldots .
$$

Thus, there exists $w^{*} \in \bar{P}_{a}$ such that $w_{n} \rightarrow w^{*}$. Applying the continuity of $T$ and $w_{n+1}=T w_{n}$, we get $T w^{*}=w^{*}$.

Let $v_{0}(t)=0,0 \leq t \leq 1$, so then $v_{0}(t) \in \bar{P}_{a}$. Let $v_{1}=T v_{0}$, so then $v_{1} \in \bar{P}_{a}$. We denote $v_{n+1}=T v_{n}=T^{n} v_{0}, n=0,1,2 \ldots$ Since $T: \bar{P}_{a} \rightarrow \bar{P}_{a}$, we have $v_{n} \in T \bar{P}_{a}$ $\subseteq \bar{P}_{a}, n=1,2, \ldots$ Since $T$ is completely continuous, we assert that $\left\{v_{n}\right\}_{n=1}^{\infty}$ is a sequentially compact set.

Since $v_{1}=T v_{0}=T 0 \in \bar{P}_{a}$,

$$
\begin{gathered}
v_{1}(t)=T v_{0}(t)=(T 0)(t) \geq 0, \quad 0 \leq t \leq 1, \\
v_{1}^{\prime}(t)=\left(T v_{0}\right)^{\prime}(t)=(T 0)^{\prime}(t) \leq 0, \quad 0 \leq t \leq 1 .
\end{gathered}
$$

So,

$$
\begin{gathered}
v_{2}(t)=T v_{1}(t) \geq(T 0)(t)=v_{1}(t), \quad 0 \leq t \leq 1, \\
v_{2}^{\prime}(t)=\left(T v_{1}\right)^{\prime}(t) \leq(T 0)^{\prime}(t)=v_{1}^{\prime}(t), \quad 0 \leq t \leq 1 .
\end{gathered}
$$

Therefore, it is similar to the earlier arguments and, by induction,

$$
v_{n+1} \geq v_{n}, \quad v_{n+1}^{\prime}(t) \leq v_{n}^{\prime}(t), \quad 0 \leq t \leq 1, n=0,1,2 \ldots
$$


Hence, there exists $v^{*} \in \bar{P}_{a}$ such that $v_{n} \rightarrow v^{*}$. Applying the continuity of $T$ and $v_{n+1}=T v_{n}$, we get $T v^{*}=v^{*}$.

If $f(t, 0,0) \not \equiv 0,0 \leq t \leq 1$, then the zero function is not the solution of (1.1) and (1.2). Thus, $\max _{0 \leq t \leq 1}\left|v^{*}(t)\right|>0$, and we have $v^{*} \geq \min \{t, 1-t\}$ $\max _{0 \leq t \leq 1}\left|v^{*}(t)\right|>0,0<t<1$.

It is well known that each fixed point of $T$ in $P$ is a solution of (1.1) and (1.2). Hence, we assert that $w^{*}$ and $v^{*}$ are two positive nonincreasing, concave solutions of the problem (1.1) and (1.2).

The proof is completed.

The following corollaries follow easily.

Corollary 3.1. Assume that (H1), (H2), (S1) and (S3) hold, and there exists a $>0$ such that:

$$
\text { (C3.1) } \begin{aligned}
\underline{\lim }_{\ell \rightarrow+\infty} \max _{0 \leq t \leq 1}\left(f(t, \ell,-a) / \ell^{p-1}\right) \leq \phi_{p}(A) \text { (in particular, } \\
\left.\underline{\lim }_{\ell \rightarrow+\infty} \max _{0 \leq t \leq 1}\left(f(t, \ell,-a) / \ell^{p-1}\right)=0\right) .
\end{aligned}
$$

Then the boundary value problem (1.1) and (1.2) has two positive, concave solutions $w^{*}$ and $v^{*}$, and all other conclusions of Theorem 3.1 hold.

Corollary 3.2. Assume that (H1), (H2) and (S3) hold, and there exist $0<a_{1}$ $<a_{2}<\cdots<a_{n}$ such that:

(C3.2.1) $f\left(t, x_{1}, y_{1}\right) \leq f\left(t, x_{2}, y_{2}\right)$ for any $0 \leq t \leq 1,0 \leq x_{1} \leq x_{2} \leq a_{k},-a_{k} \leq y_{2}$ $\leq y_{1} \leq 0, k=1,2, \ldots, n$;

(C3.2.2) $\max _{0 \leq t \leq 1} f\left(t, a_{k},-a_{k}\right) \leq \phi_{p}\left(a_{k} A\right), k=1,2, \ldots, n$.

Then the boundary value problem (1.1) and (1.2) has $2 n$ positive, concave solutions $w_{k}^{*}$ and $v_{k}^{*}$ such that:

$$
\begin{aligned}
& \quad 0<w_{k}^{*} \leq a_{k}, \quad-a_{k}<\left(w_{k}^{*}\right)^{\prime} \leq 0, \\
& \text { and } \quad \lim _{n \rightarrow \infty} w_{k_{n}}=\lim _{n \rightarrow \infty} T^{n} w_{k_{0}}=w_{k}^{*}, \quad \lim _{n \rightarrow \infty}\left(w_{k_{n}}\right)^{\prime}=\lim _{n \rightarrow \infty}\left(T^{n} w_{k_{0}}\right)^{\prime}=\left(w_{k}^{*}\right)^{\prime}, \\
& \text { where } \quad w_{k_{0}}(t)=a_{k}-a_{k} t \frac{1-\sum_{i=1}^{n} \beta_{i}}{1-\sum_{i=1}^{n} \beta_{i} \xi_{i}}, \quad 0 \leq t \leq 1,
\end{aligned}
$$

and

$$
\begin{aligned}
& \quad 0<v_{k}^{*} \leq a_{k}, \quad-a_{k}<\left(v_{k}^{*}\right)^{\prime} \leq 0, \\
& \text { and } \lim _{n \rightarrow \infty} v_{k_{n}}=\lim _{n \rightarrow \infty} T^{n} v_{k_{0}}=v_{k}^{*}, \quad \lim _{n \rightarrow \infty}\left(v_{k_{n}}\right)^{\prime}=\lim _{n \rightarrow \infty}\left(T^{n} v_{k_{0}}\right)^{\prime}=\left(v_{k}^{*}\right)^{\prime}, \\
& \text { where } v_{k_{0}}(t)=0, \quad 0 \leq t \leq 1,
\end{aligned}
$$

with $(T u)(t)$ defined in the same way as in (3.1). 
The iterative schemes in Corollary 3.2 are

$$
\begin{aligned}
w_{k_{0}}(t)= & a_{k}-a_{k} t \frac{1-\sum_{i=1}^{n} \beta_{i}}{1-\sum_{i=1}^{n} \beta_{i} \xi_{i}}, \quad w_{k_{n+1}}=T w_{k_{n}} \\
= & T^{n} w_{k_{0}}, \quad k=1,2 \ldots, n=0,1,2 \ldots \\
& \quad \text { and } \quad v_{k_{0}}(t)=0, \quad v_{k_{n+1}}=T v_{k_{n}}=T^{n} v_{k_{0}}, \quad k=1,2 \ldots, n=0,1,2 \ldots
\end{aligned}
$$

They start off with known linear functions and zero functions respectively.

Corollary 3.3. Assume that (H1), (H2), (C3.2.1) and (S3) hold, and there exist $0<a_{1}<a_{2}<\cdots<a_{n}$ such that

(C3.3) $\underline{\lim }_{\ell \rightarrow+\infty} \max _{0 \leq t \leq 1}\left(f\left(t, \ell,-a_{k}\right)\right) /\left(\ell^{p-1}\right) \leq \phi_{p}(A), k=1,2, \ldots, n$ (in particular, $\left.\underline{\lim }_{\ell \rightarrow+\infty} \max _{0 \leq t \leq 1}\left(f\left(t, \ell,-a_{k}\right) / \ell^{p-1}\right)=0, k=1,2, \ldots, n\right)$.

Then the boundary value problem (1.1) and (1.2) has $2 n$ positive, concave solutions $w_{k}^{*}$ and $v_{k}^{*}$, and all other conclusions of Corollary 3.2 hold.

\section{Acknowledgements}

This work was sponsored by the National Natural Science Foundation of China (10671012) and the Doctoral Program Foundation of Education Ministry of China (20050007011).

\section{References}

[1] W. Feng, "On an $m$-point boundary value problem", Nonlinear Anal. 30 (1997) 5369-5374.

[2] W. Feng and J. Webb, "Solvability of $m$-point boundary value problem with nonlinear growth", J. Math. Anal. Appl. 212 (1997) 467-480.

[3] C. Gupta, "A generalized multipoint boundary value problem for second order ordinary differential equations", Appl. Math. Comput. 89 (1998) 133-146.

[4] V. II'in and E. Moiseev, "Nonlocal boundary value problem of the second kind for a SturmLiouville operator", Differ. Equ. 23 (1987) 979-987.

[5] R. Ma, "Existence of solutions of nonlinear $m$-point boundary value problem", J. Math. Anal. Appl. 256 (2001) 556-567.

[6] R. Ma, "Multiple positive solutions for nonlinear $m$-point boundary value problems", Appl. Math. Comput. 148 (2004) 249-262.

[7] D. Ma, Z. Du and W. Ge, "Existence and iteration of monotone positive solutions for multipoint boundary value problems with $p$-Laplacian operator", Comput. Math. Appl. 50 (2005) 729-739.

[8] Y. Wang, "Existence of multiple positive solutions for one-dimensional $p$-Laplacian", J. Math. Anal. Appl. 315 (2006) 144-153. 